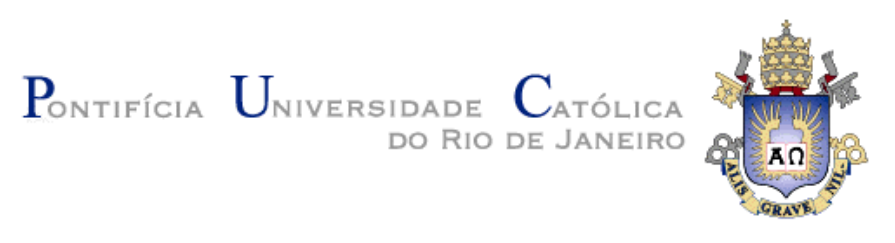

Jordan Janeiro Lopes da Silva

\title{
Um Protocolo Sensível ao Contexto para Adaptação Coordenada de Serviços de Comunicação em Redes sem
}

Fio

\section{Dissertação de Mestrado}

Dissertação apresentada como requisito parcial para obtenção do título de Mestre pelo Programa de PósGraduação em Informática da PUC-Rio.

Orientador: Markus Endler

Rio de Janeiro

Abril de 2008 


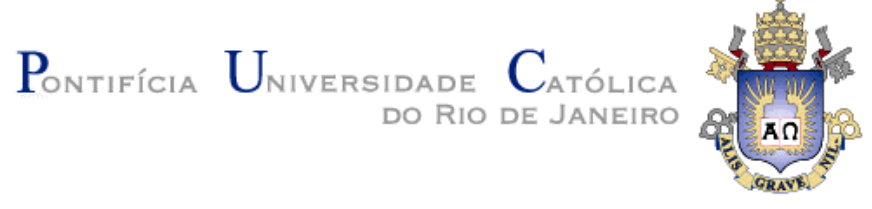

Jordan Janeiro Lopes da Silva

\title{
Um Protocolo Sensível ao Contexto para Adaptação Coordenada de Serviços de Comunicação em Redes sem
}

Fio

Dissertação apresentada como requisito parcial para obtenção do título de Mestre pelo Programa de PósGraduação em Informática da PUC-Rio. Aprovada pela Comissão Examinadora abaixo assinada.

\author{
Markus Endler \\ Orientador \\ Departamento de Informática - PUC-Rio \\ Noemi Rodriguez \\ Departamento de Informática - PUC-Rio \\ Antônio Tadeu Azevedo Gomes \\ LNCC \\ Renato Fontoura de Gusmão Cerqueira \\ Departamento de Informática - PUC-Rio \\ José Ferreira de Rezende \\ Departamento de Ciência da Computação - UFRJ \\ José Eugênio Leal
}

Coordenador(a) Setorial do Centro Técnico Científico - PUC-Rio

Rio de Janeiro

24 de Abril de 2008 
Todos os direitos reservados. É proibida a reprodução total ou parcial do trabalho sem autorização da universidade, do autor e do orientador.

\section{Jordan Janeiro Lopes da Silva}

Bacharel em Informática pela Pontifícia Universidade Católica do Rio de Janeiro (PUC-Rio) em 2005. Atualmente integra a equipe do LAC desenvolvendo pesquisa em Computação Móvel.

Ficha Catalográfica

Silva, Jordan

Um Protocolo Sensível ao Contexto para Adaptação Coordenada de Serviços de Comunicação em Grupos I Jordan Janeiro Lopes da Silvar; orientador: Markus Endler. - Rio de Janeiro: PUC, Departamento de Informática, 2008.

96 f. : il. (col.) ; 29,7 cm

Dissertação (Mestrado) - Pontifícia Universidade Católica do Rio de Janeiro, Departamento de Informática.Rio de Janeiro, 2008.

Incluí referências bibliográficas.

1. Informática - Teses. 2. Contexto. 3. Contexto Global. 4. Adaptação Dinâmica Sensível ao Contexto. 5. Adaptação Transparente. 6. Tolerância à Desconexões. 7. Redes Sem Fio. 8. Comunicação em Grupo Confiável I. Endler, Markus. II. Pontifícia Universidade Católica do Rio de Janeiro. Departamento de Informática. III. Título. 


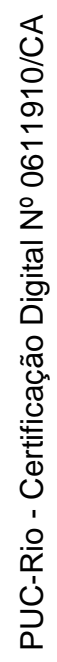

à Nilva, Fátima e Renan. 


\section{Agradecimentos}

Agradeço ao Grande Arquiteto do Universo concluir mais uma jornada na minha vida.

Agradeço ao meu orientador Markus Endler pela paciência, compreensão e pela confiança depositada em mim.

Agradeço à minha família: Nilva (avó), Fátima (mãe) e Renan (irmão) pelo incentivo e comprenssão em todas as minhas decisões. Agradeço também pelos finais de semana mais "comportados" que precisei para trabalhar.

Agradeço aos irmãos que escolhi: Bruno e Gustavo, que já me acompanham há 14 anos. Sem eles a vida não seria tão "pós-moderna".

Agradeço à Renata pelo apoio nos momentos mais críticos deste trabalho, e sua família por vezes me receber tarde da noite.

Agradeço a todos os meus companheiros do LAC: Gustavo, Hana, Hubert e Juliana. Em especial Viterbo, pelas conversas e trocas de idéias, Ricardo, pelos conselhos e ajuda, e Malcher pelo companherismo. 


\section{Resumo}

Silva, Jordan; Endler, Markus. Um Protocolo Sensível ao Contexto para Adaptação Coordenada de Serviços de Comunicação em Redes sem Fio. Rio de Janeiro, 2008. 96p. Dissertação de Mestrado - Departamento de Informática, Pontifícia Universidade Católica do Rio de Janeiro.

A pesquisa em redes móveis e computação pervasiva têm demonstrado que adaptação dinâmica e sensibilidade ao contexto são requisitos básicos para aplicações nestes ambientes. Muitos dos trabalhos sobre adaptação dinâmica sensível ao contexto encontrados na literatura se baseiam nas informações de contexto de dispositivos para selecionar e executar uma determinada adaptação, mas somente no próprio dispositivo. Para aplicações distribuídas cientes do contexto envolvendo um grupo de dispositivos portáteis, executando em uma rede sem fio, por vezes faz-se necessária uma adaptação conjunta e coordenada de todos os dispositivos do grupo, adaptação essa que depende de um contexto global do grupo. Este trabalho apresenta Moratus, um protocolo para obter o contexto global de um grupo de dispositivos e executar uma única adaptação de serviços de comunicação em todos os dispositivos de forma coordenada. Este protocolo também leva em conta e trata desconexões involuntárias de membros do grupo durante o processo. O Moratus é o elemento central de um middleware, o SACS, que permite que tal adaptação coordenada ocorra de forma transparente e sem interrupção para a aplicação distribuída que usa o middleware.

\section{Palavras-chave}

Contexto; Contexto Global; Adaptação Dinâmica Sensível ao Contexto; Adaptação Transparente; Tolerância à Desconexões; Redes Sem Fio; Comunicação em Grupo Confiável 


\section{Abstract}

Silva, Jordan; Endler, Markus. A Context-Aware Protocol for Coordinated Adaptation of Communication Services in Wireless Networks. Rio de Janeiro, 2008. 96 p. Master Thesis - Departamento de Informática, Pontifícia Universidade Católica do Rio de Janeiro.

Research in mobile networks and pervasive computing has shown that dynamic adaptation and context-awareness are basic requirements for applications executing in such environments. Many of the works about context-awareness dynamic adaptation found in the literature use the device's context information to execute an adaptation only locally at the device. For distributed, context-aware applications composed of a group of portable devices (executing in a wireless network) sometimes it is necessary to perform a collective and coordinated adaptation at all the devices of the group, and where this adaptation depends on the global context of the group. This thesis presents Moratus, a protocol that processes the global context of a group and executes a unique adaptation of a communication service at all devices in the group in a coordinated way. This protocol also handles unplanned disconnections of group members during the adaptation process. Moratus is the central element of a middleware named SACS, which allows that such coordinated adaptation is performed transparently and without disruption for the distributed application based on this middleware.

\section{Keywords}

Context; Global Context; Context-Awareness Dynamic Adaptation; Transparent Adaptation; Disconnection Tolerance; Wireless Networks; Reliable Group Communication 


\section{Sumário}

1 Introdução 14

1.1. Objetivos 15

1.2. Organização da Dissertação 16

2 Fundamentação Conceitual 17

2.1. Contexto Local 17

2.2. Contexto Global 18

2.2.1. Cenários de Uso 18

2.2.1.1. Nível de Energia Global 19

2.2.1.2. Intensidade do Sinal de Radiofreqüência Global 19

2.3. Gerenciamento de Grupos 20

2.4. Serviços de Transformação de Mensagens 21

3 Moratus $\quad 22$

3.1. Etapas do Protocolo 23

3.1.1. Eleição do Contexto Global 24

3.1.2. Adaptação de Serviços de Transformação de Mensagens 26

3.2. Serviço de Adaptação Coordenada de Serviços de

Transformação de Mensagens 28

3.2.1. Arquitetura 29

3.2.2. Serviços de Apoio ao Moratus 32

4 Implementação 34

4.1. Obtenção de Contexto 34

4.2. Camada de Comunicação de Grupo 35

4.3. Eleição do Contexto Global 39

4.3.1. Convocação da Eleição do Contexto Global 39

4.3.2. Recebimento de Contexto 41

4.3.3. Tratamento de Desconexão 43

4.4. Adaptação de Serviços de Transformação de Mensagens 44 
4.4.1. Instalação de Serviços 44

4.4.2. Finalização da Adaptação de Serviços 46

4.4.3. Tratamento de Desconexão 47

5 Avaliação 49

5.1. Testes de Desempenho 49

5.1.1. Testes na Rede Cabeada 50

5.2. Testes de Desconexão 53

6 Trabalhos Relacionados $\quad 62$

6.1. Critérios Comparativos 62

6.2. Ensemble 62

6.2.1. Arquitetura do Ensemble 64

6.2.2. Protocolo de Troca de Protocolo 65

6.3. Processo de Adaptação Graciosa 68

6.3.1. Arquitetura 68

6.3.2. Técnicas de Adaptação 70

6.3.2.1. Detecção de Mudanças 70

6.3.2.2. Consenso 71

6.3.2.3. Ação da Adaptação 72

6.3.2.4. Interface dos Módulos de Algoritmos de Adaptação $\begin{array}{ll}\text { Alternativos } & 74\end{array}$

6.4. NeCoMan: Network Consistency Management 74

6.4.1. Processo de Adaptação Segura de Serviços Distribuídos 75

6.4.1.1. Instalação de Novos Serviços 76

6.4.1.2. Ativação do Novo Serviço 76

6.4.1.3. Remoção do Antigo Serviço 77

6.4.2. Reflexividade $\quad 77$

6.4.2.1. Modelo de Comunicação do Serviço 78

6.4.2.2. Embaralhamento de Pacotes 80

6.4.2.3. Transferência de Estado 80

6.5. Estudo Comparativo 81

6.5.1. Transparência da Adaptação 81

6.5.2. Tratamento da Desconexão 81 
6.5.3. Composição de Pilhas de Protocolos 82

6.5.4. Coordenação da Adaptação Distribuída 83

7 Conclusão $\quad 84$

7.1. Trabalhos Futuros 85

8 Bibliografia $\quad 88$

$\begin{array}{ll}\text { Apêndice A - Tipos de Mensagens } & 91\end{array}$ 


\section{Lista de figuras}

Figura 1 - Casos de uso da fase de eleição do contexto global. 24

Figura 2 - Utilização do Serviço de Middleware SACS. 28

Figura 3 - Arquitetura do Serviço de Adaptação Coordenada de Serviços de Transformação de Mensagens (SACS). 29

Figura 4 - Arquitetura do JGroups. 36

Figura 5 - Convocação da Eleição do Contexto Global. 40

Figura 6 - Receber contexto e responder a convocação da eleição do contexto global.

Figura 7 - Tratamento da desconexão de um dispositivo na etapa de eleição do contexto global. 43

Figura 8 - Instalação de um serviço de transformação de mensagens. 45

Figura 9 - Finalização da etapa de adaptação de serviços de transformação de mensagens e do processo adaptativo. 46

Figura 10 - Tempo decorrido no processo adaptativo para grupos de diferentes tamanhos.

Figura 11 - Tempo de execução da etapa de adaptação de serviços de transformação de mensagens.

Figura 12 - Tempos de execução de cada uma das fases da etapa de adaptação de serviços.

Figura 13 - Desconexão de um dispositivo na etapa de eleição do contexto global.

Figura 14 - Desconexão do coordenador na etapa de adaptação de serviços de transformação de mensagens.

Figura 15 - Desconexão do participante na etapa de adaptação de serviços de transformação de mensagens.

Figura 16 - Retransmissão da Mensagem de Adaptação entre Camadas.

Figura 17 - Uma amostra dos eventos mais comuns de serem transitados para cima e para baixo na pilha de protocolos.

Figura 18 - Arquitetura do Ensemble.

Figura 19 - O protocolo de troca de protocolos. 
Figura 20 - Arquitetura do sistema no processo de adaptação graciosa. $\quad 69$

$\begin{array}{ll}\text { Figura } 21 \text { - Estrutura de um componente adaptável. } & 70\end{array}$

Figura 22 - Processo de Adaptação. $\quad 74$

Figura 23 - Algoritmo do processo de adaptação segura de serviços. 75

Figura 24 - Modelo genérico do processo de instalação de serviços. 78

Figura 25 - Modelos de comunicação entre os nós de um serviço. 78

Figura 26 - Instâncias personalizadas do modelo genérico da instalação de um novo serviço. 


\section{Lista de tabelas}

Tabela 1 - Definição dos intervalos da variação da intensidade do sinal.

Tabela 2 - Log do tratamento de desconexão de um dispositivo na etapa de eleição do contexto global.

Tabela 3 - Log do tratamento de desconexão do coordenador na etapa de adaptação de serviços de transformação de mensagens.

Tabela 4 - Log do tratamento de desconexão do participante na etapa de adaptação de serviços de transformação de mensagens.

Tabela 5 - Tempos de execução da etapa de eleição do contexto global, incluindo o tempo do tratamento da desconexão de um dispositivo.

Tabela 6 - Tempos de execução da etapa de adaptação de serviços de transformação de mensagens, incluindo o tempo do tratamento da desconexão de um dispositivo.

Tabela 7 - Comparação entre os trabalhos relacionados ao Moratus. 